\title{
Novel mutation in AAA domain of BCS1L causing Bjornstad syndrome
}

\author{
Saima Siddiqi ${ }^{1}$, Saadat Siddiq ${ }^{2}$, Atika Mansoor ${ }^{1}$, Jaap Oostrik ${ }^{3,4,5}$, Nafees Ahmad ${ }^{1}$, Syed Ali Raza Kazmi ${ }^{1}$, \\ Hannie Kremer ${ }^{3,4,5,6}$, Raheel Qamar ${ }^{7,8}$ and Margit Schraders $3,4,5$
}

Bjørnstad syndrome is an extremely rare condition characterized by pilitorti and nerve deafness. Only few large families have been reported worldwide. Here we describe a large Pakistani family with five affected individuals. The hair fibers of all the patients were twisted around their axis and devoid of any pigment. In addition the patients had a moderate-to-severe degree of hearing impairment. Genotyping with high-density single-nucleotide polymorphism arrays showed homozygosity in two intervals on chromosome 2. Linkage with one of these regions (genomic position 218745685-221025443, hg19) was confirmed. This region encompasses the $B C S 1 L$ gene. Mutations in this gene have previously been associated with Bjørnstad's syndrome. We sequenced the $B C S 1 L$ gene for identification of the causative mutation in the family. A novel homozygous missense mutation c.901T $>$ A was identified, which segregated with the disease in the family. This mutation results in the amino acid change p.Tyr301Asn and was predicted to be pathogenic by bioinformatics tools.

Journal of Human Genetics (2013) 58, 819-821; doi:10.1038/jhg.2013.101; published online 31 October 2013

Keywords: BCS1L; Bjørnstad's syndrome; pilitorti; sensorineural hearing loss

Bjørnstad syndrome is a rare inherited autosomal recessive disorder (BJS, OMIM \#603647), characterized by pilitorti and nerve deafness, which was first described by Bjørnstad. ${ }^{1}$ Previously, only one large family with an autosomal recessive inheritance has been described by Lubianca et al. ${ }^{2}$

In the current study, we describe a large consanguineous family from Pakistan with five affected members, three males and two females (Figure 1a). The patients had scalp hair at birth that along with the eyelashes began falling out at the age of 2-3 months, eventually leaving only sparse short hair growth over the scalp and the rest of the body (Figure 1b). The hair fibers of all the patients were twisted around their axis at an angle of $180^{\circ}$ (Figure 1c) and were devoid of any pigment. In addition, the eyes of the affected males and females were of light color and all the patients had anhidrosis. Teeth, nails, palms and soles were normal in all the affected individuals. All affected males were short in stature and had lean bodies (height, $156.3 \pm 4.5 \mathrm{~cm}$; weight, $40.3 \pm 4.5 \mathrm{~kg}$ ) as compared with the normal males of the family (height, $176 \pm 5 \mathrm{~cm}$ weight, $62 \pm 4 \mathrm{~kg}$; Figure 1d). Affected male members were fertile, thus excluding the possibility of hypogonadism, which is characteristic of Crandall syndrome. Audiometric measurements revealed various degrees of bilateral sensorineural hearing loss, moderate to severe in children and sever to profound in adult patients showing the progressive hearing loss (Table 1).
All the affected family members were genotyped using Illumina Human Omni Express 700K arrays according to the manufacturer's instructions. Homozygosity Mapper was used to find the homozygous regions shared by all affected individuals (www.homozygositymapper.org). Two regions on chromosome 2 were identified; chr2:162785323-177648762 bp (hg19) flanked by rs10930036 and rs10177590, and chr2:221025443-218745685 bp (hg19), which was flanked by rs10199795 and rs10205602. The first region was excluded by sequence tagged sites (STS) markers. The 2 nd region encompassed 2.2 $\mathrm{Mb}$ and encompassed 72 annotated genes including the BCS1L gene, known to be associated with Bjørnstad syndrome. ${ }^{3}$ Therefore, we first analyzed this gene for the presence of any possible mutation responsible for Bjørnstad syndrome in this family.

All coding exons and exon-intron boundaries of $B C S 1 L$ (NM_004324) were sequenced using primers (Table 2) designed from Exon Primers (UCSC; http://genome.ucsc.edu/). A novel variant c.901T $>A$ was identified in exon 8, predicted to cause an amino acid change, p.Tyr301Asn (Figure 2a and c). Previously, five different mutations in this gene have been reported to be associated with pilitorti and sensorineural deafness in familial or isolated cases (Swissprot variant database; http://swissvar.expasy.org/). Residue Tyr301 and the Tyr301Asn mutation are located within the topological domain on the surface of the protein and the substitution results in a decrease in hydrophobic interactions with other molecules as

\footnotetext{
${ }^{1}$ Institute of Biomedical and Genetic Engineering (IBGE), Islamabad, Pakistan; ${ }^{2}$ National Institute of Handicap, Islamabad, Pakistan; ${ }^{3}$ Department of Otorhinolaryngology, Head and Neck Surgery, Nijmegen, The Netherlands; ${ }^{4}$ Nijmegen Centre for Molecular Life Sciences, Nijmegen, The Netherlands; ${ }^{5}$ Donders Institute for Brain, Cognition and Behaviour, Nijmegen, The Netherlands; ${ }^{6}$ Department of Human Genetics, Radboud University Nijmegen Medical Centre, Nijmegen, The Netherlands; ${ }^{7}$ COMSATS Institute of Information Technology, Islamabad, Pakistan and ${ }^{8} \mathrm{Al}$-Nafees Medical College and Hospital, Isra University, Islamabad, Pakistan Correspondence: Dr S Siddiqi, Institute of Biomedical and Genetic Engineering (IBGE), Islamabad., 24-Mauve area, G-9/1, Islamabad 44000, Pakistan. E-mail: saimasiddiqi2@gmail.com
}

Received 28 February 2013; revised 19 June 2013; accepted 1 August 2013; published online 31 October 2013 


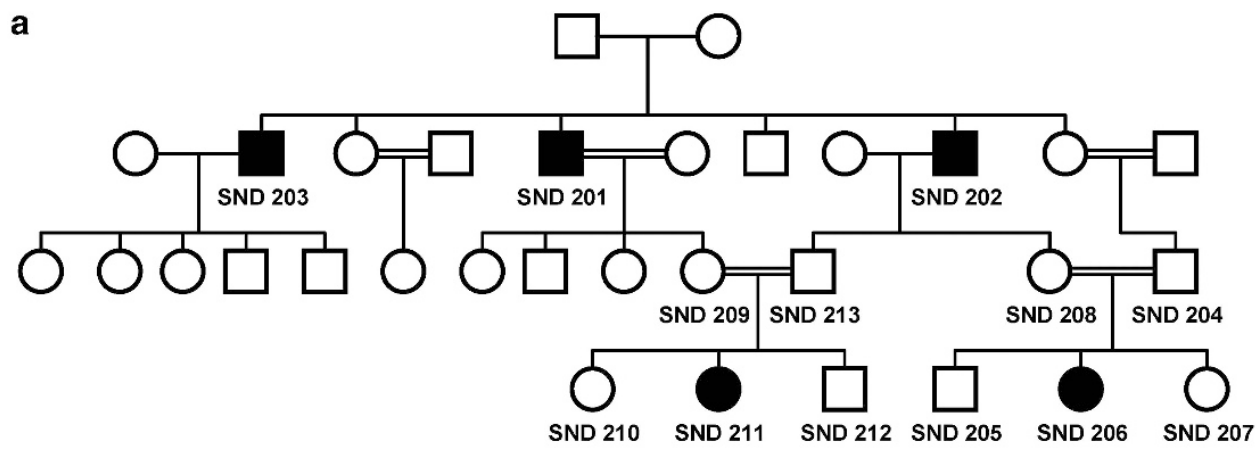

b

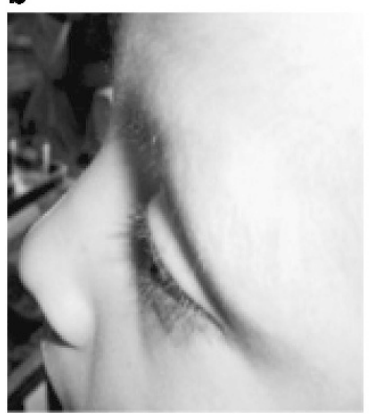

C

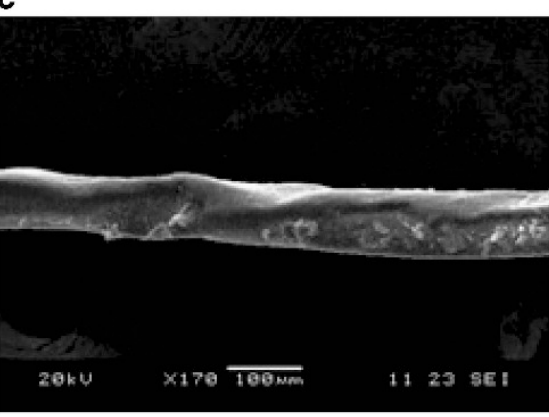

d

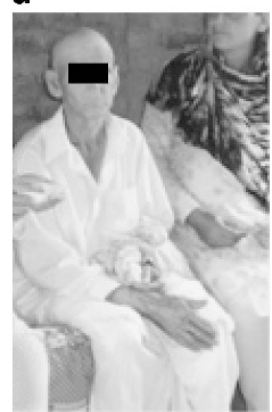

Figure 1 (a) Family pedigree showing an autosomal recessive mode of inheritance with pilitorti and nerve deafness. (b) A young patient showing hair loss from the scalp and eyelashes. (c) Scanning electron microscopic scan of a hair fiber of patient II:5 showing multiple twists of $180^{\circ}$. (d) An affected male with short stature as compared to a normal female of the family. A full color version of this figure is available at the Journal of Human Genetics journal online.

Table 1 Clinical features of all affected family members

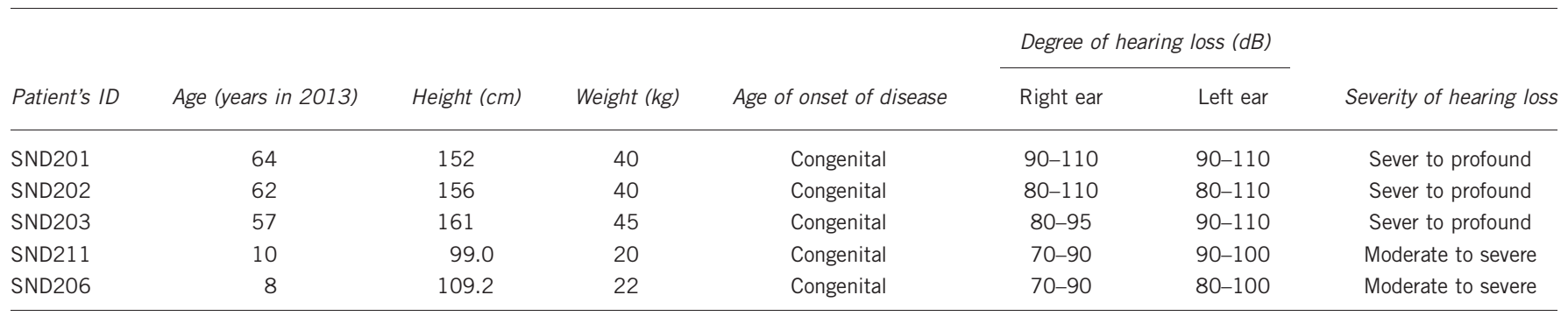

Table 2 Primer sequences used to amplify and sequence the exons of the BCS1L gene

\begin{tabular}{llc}
\hline Primer name & Sequence & Product size \\
\hline BCS1L_Ex3_Fw & CACCCCTAGGTTTTCGTAAC & 452 \\
BCS1L_Ex3_Rev & GTGAGTGAATGGGTCAAACC & \\
BCS1L_Ex4_Fw & GAAAAGAATGATGGGAGCTG & 268 \\
BCS1L_Ex4_Rev & ATCTGATGTCAAATGTCCCC & \\
BCS1L_Ex5_Fw & CCAGAAGGAAGCTGTTTGG & 373 \\
BCS1L_Ex5_Rev & TATATCACCCCATCTGGCTG & \\
BCS1L_Ex6_7_Fw & CAGGGCTGGGATGAAC & 619 \\
BCS1L_Ex6_7_Rev & CCCTCTGGATTTCTGCTTC & \\
BCS1L_Ex8_Fw & AGTAGGGGAACATAGTGGGG & 255 \\
BCS1L_Ex8_Rev & CACTAGGGGAGGAGGTGG & \\
BCS1L_EX9_Fw & CTTGGGAGGAACAGGAGG & 391 \\
BCS1L_Ex9_Rev & GCAGAGTAGTGTGGCAGATG & \\
\hline
\end{tabular}

predicted by PROJECT HOPE (http://www.cmbi.ru.nl/hope; Figure $2 b) .{ }^{4}$ The mutation segregates with the disease in the family and was neither found in 137 Pakistani unaffected control individuals, as analyzed by restriction fragment length polymorphism (Supplementary Figure S2) nor in the 1000-genome data (http:// evs.gs.washington.edu).

BCS1L encodes a homolog of the Saccharomyces cerevisiae bcs1 protein, which is involved in the assembly of complex III of the mitochondrial respiratory chain. The BCS1L protein has two domains, the BCS domain and the AAA ATPase type core. ${ }^{3}$ Two alternatively spliced transcripts encoding the same protein have been described. The AAA ATPase core is present from amino acid 222 to 357 (prediction by SMART) and 19 different mutations have been described in this domain (Figure 2c), resulting in heterogenous phenotypes ranging from lethal Gracile syndrome or complex III deficiency to milder disorders such as Bjørnstad syndrome (Swissprot variant database). The hydrophobic changes in the AAA domain due 
a
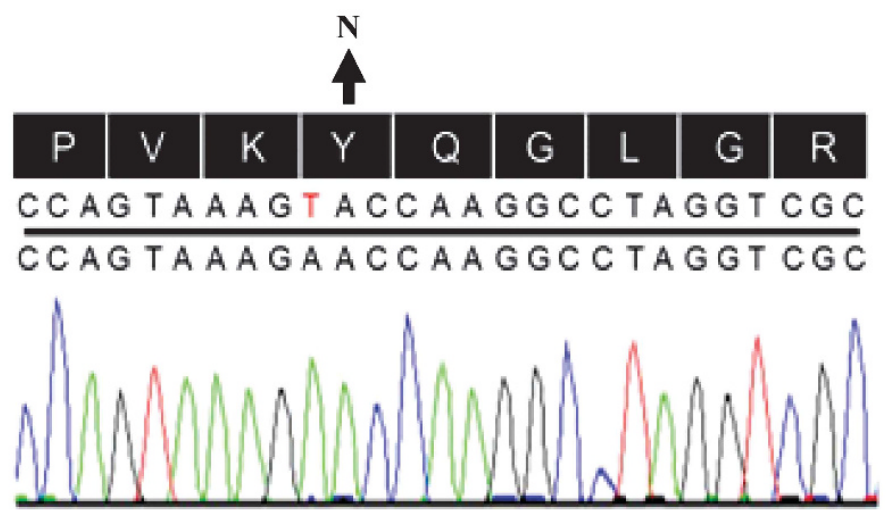

b

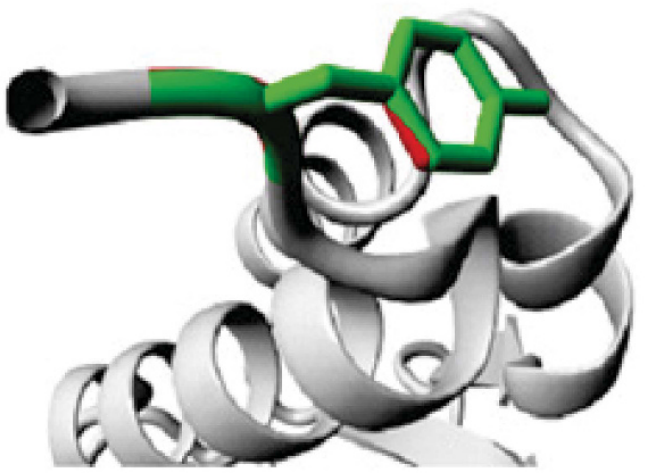

C
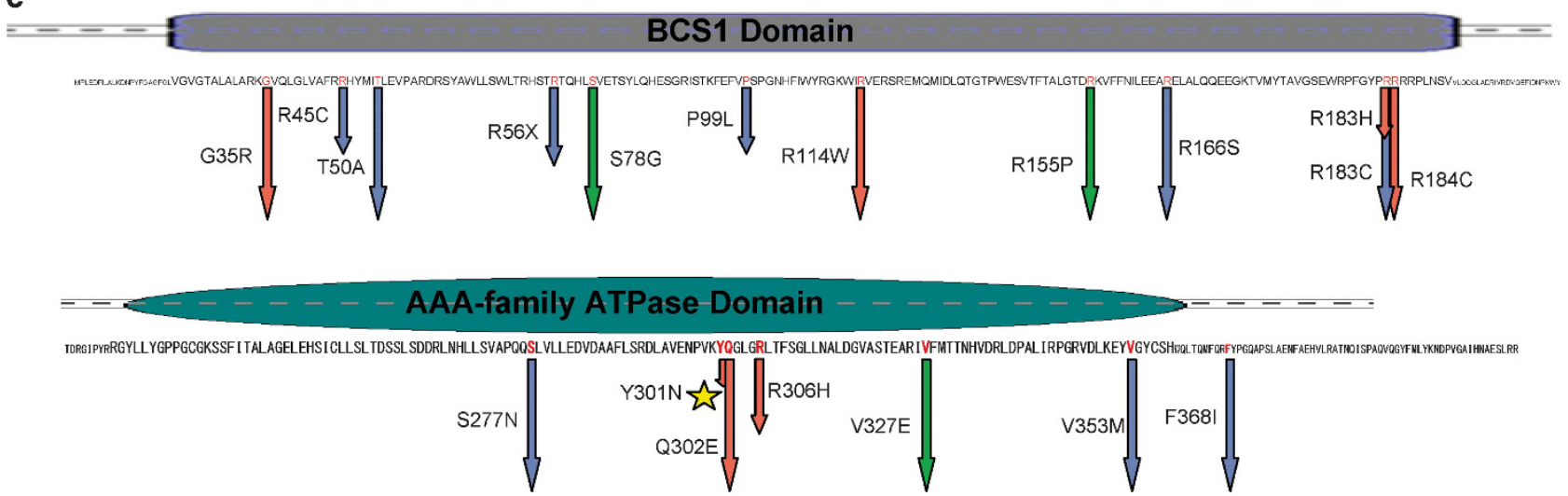

Figure 2 (a) Sequence analysis of patient showing c.901T>A change (g.219,527,617). The upper sequence is the reference sequence representing the mutation site shown in red color. (b) Location of the mutation in part of the three-dimensional structure of the BCS1L protein. The mutation results in a change in the structure of the protein. The green residue represents the normal tyrosine; the red residue represents the mutant asparagine. This mutation is present in the ATPase domain of the BCS1L gene (c). All mutations reported so far in the BCS1L gene and the encoded protein domains are represented in graphical form of the protein (c). Orange arrows indicate the mutations for Bjørnstad syndrome, blue arrows mark the mutations responsible for mitochondrial complex III deficiency and pink arrows for mutations in Gracile syndrome. The asterisk shows the novel Tyr301Asn mutation identified in the present study.

to the mutation described in the present study can result in changing the binding affinity of this domain for effector and other molecules. The reduction in binding affinity disrupts the complex III assembly, which in turn reduces the activity of the electron transport chain resulting in the disease pathology. This observation is supported by previous studies also, where amino-acid residues at position 302 and 306 have also been shown to cause Bjørnstad syndrome and can provide further insights into the pathogenic role of BCS1L in mitochondrial function, which will result in a better understanding of the disease pathology and improved management of the disease.

\section{ACKNOWLEDGEMENTS}

We are thankful to all the family members for their participation in the study. We also thank Dr Jamsheed-bin-Saeed, ENT Specialist at Maroof Int. Hospital,
Islamabad, and Dr Ammad-ul-Hasan, audiologist at GMT Islamabad, for conducting the clinical tests. The project work was supported in part by ZonMW grant 40-00812-98-09047 and PAS/I-9/Project award (RQ).

1 Bjørnstad, R. Pilitorti and sensory-neural loss of hearing. Proc. Fenno-Scand. Ass. Derm. 3-12 (1965).

2 Lubianca, N. J. F., Lu, L., Eavey, R. D., Flores, M. A., Caldera, R. M., Sangwatanaroj, S. et al. The Bjornstad syndrome (sensorineural hearing loss and pili torti) disease gene maps to chromosome 2q34-36. Am. J. Hum. Genet. 62, 1107-1112 (1998).

3 Hinson, J. T., Fantin, V. R., Schonberger, J., Breivik, N., Siem, G., McDonough, B. et al. Missense mutations in the BCS1L gene as a cause of the Bjornstad syndrome. N. Engl. J. Med. 356, 809-819 (2007).

4 Venselaar, H., Te Beek, T. A., Kuipers, R. K., Hekkelman, M. L. \& Vriend, G. Protein structure analysis of mutations causing inheritable diseases. An e-Science approach with life scientist friendly interfaces. BMC Bioinformatics. 11, 548 (2010).

Supplementary Information accompanies the paper on Journal of Human Genetics website (http://www.nature.com/jhg) 